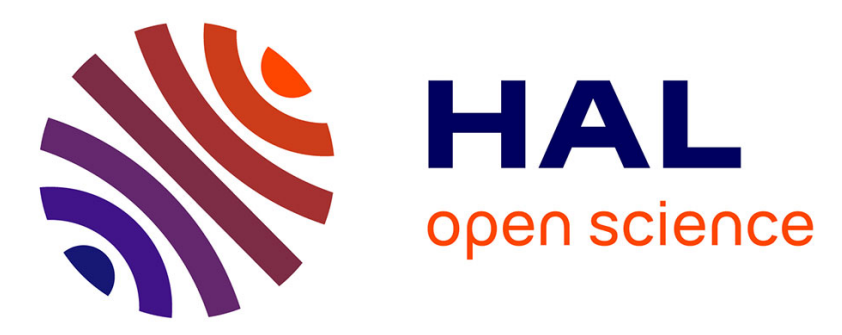

\title{
Manufacturability analysis to combine additive and subtractive processes
}

\author{
Olivier Kerbrat, Pascal Mognol, Jean-Yves Hascoët
}

\section{To cite this version:}

Olivier Kerbrat, Pascal Mognol, Jean-Yves Hascoët. Manufacturability analysis to combine additive and subtractive processes. Rapid Prototyping Journal, 2010, 16 (1), pp.63-72. 10.1108/13552541011011721 . hal-00522068

\section{HAL Id: hal-00522068 \\ https://hal.science/hal-00522068}

Submitted on 29 Sep 2010

HAL is a multi-disciplinary open access archive for the deposit and dissemination of scientific research documents, whether they are published or not. The documents may come from teaching and research institutions in France or abroad, or from public or private research centers.
L'archive ouverte pluridisciplinaire $\mathbf{H A L}$, est destinée au dépôt et à la diffusion de documents scientifiques de niveau recherche, publiés ou non, émanant des établissements d'enseignement et de recherche français ou étrangers, des laboratoires publics ou privés. 


\title{
MANUFACTURABILITY ANALYSIS TO COMBINE ADDITIVE AND SUBTRACTIVE PROCESSES
}

\author{
Authors: Olivier Kerbrat, Pascal Mognol, Jean-Yves Hascoët \\ IRCCyN (Institut de Recherche en Communications et Cybernétique de Nantes) \\ 1 rue de la Nö̈-BP 92101 - F-44321 Nantes Cedex 03, Nantes, France \\ Corresponding author: Olivier Kerbrat \\ IRCCyN / MO2P team \\ 1 rue de la Noë \\ BP 92101 \\ F-44321 Nantes Cedex 03 \\ France \\ Tel.: +33(0)299055275 \\ Fax: +33(0)299059328 \\ E-mail address: Olivier.Kerbrat@irccyn.ec-nantes.fr
}

\section{Purpose:}

The aim of this paper is to propose a methodology to estimate manufacturing complexity for both machining and layered manufacturing. The goal is to take into account manufacturing constraints at design stage in order to realize tools (dies and molds) by a combination of a subtractive process (HighSpeed Machining) and an additive process (Selective Laser Sintering).

\section{Design/methodology/approach:}

Manufacturability indexes are defined and calculated from the tool CAD model, according to geometric, material and specification information. The indexes are divided into two categories: global and local. For local indexes, a decomposition of the tool CAD model is used, based on an octree decomposition algorithm, and a map of manufacturing complexity is obtained.

\section{Findings:}

The manufacturability indexes values provide a well detailed view of which areas of the tool may advantageously be machined or manufactured by an additive process.

\section{Originality/value:}

Nowadays, layered manufacturing processes are coming to maturity, but there are still no way to compare these new processes with traditional ones (like machining) at the early design stage. In this paper, a new methodology is proposed to combine additive and subtractive processes, for tooling design and manufacturing. A manufacturability analysis is based on an octree decomposition, with calculation of manufacturing complexity indexes from the tool CAD model. 


\section{Context of the study: hybrid modular tooling}

In order to improve competitiveness in modern mass production industry, products have to be designed and manufactured with the following two goals that are often in opposition:

- Decreasing time and cost;

- Improving quality and flexibility.

These objectives imply two design and manufacturing constraints: a rapid manufacturing and a high level of reactivity when design evolutions are required. The current field of tooling (dies and molds) does not break these constraints and one answer to the problem is to design and manufacture hybrid modular tools, with modular and hybrid points of views.

Modular point of view: Instead of a single-piece tool, it is seen as a 3-D puzzle with modules realized separately and further assembled. The two advantages are: each module may be produced simultaneously and few modules may be changed without changing the whole tool. With this point of view, several alternatives of the same product may advantageously be manufactured with the same mold, changing one module to provide new part functions.

Hybrid point of view: Each module of the tool is manufactured by the best process, in term of time, cost and/or quality. Presently, focus is put on comparison between a subtractive process (HSM: High-Speed Machining) and an additive process (SLS: Selective Laser Sintering). Another research topic investigates the combination of these two manufacturing processes.

To illustrate the advantages of using hybrid modular tooling, Figure 1 presents an industrial example, developed at IRCCyN (Mognol et al., 2007). It is a part from automotive industry, manufactured by injection molding (Figure 1). In this part, the positions of the circled shapes have diversifying alternatives, there are a marking that changes with the model and an evolutionary feature for the seal positioning (Figure 1(b)). The part with its evolutionary areas may be produced with just one mold creating modules for each changing area of the piece. So modules are designed (Figure 1(c)) and realized with the best process (Figure 1(d)), and changed when the product model is modified.

Take in Figure 1.

\section{Manufacturability analysis issue for hybrid modular tooling}

The two points of views, hybrid and modular, have allowed creating rapid tools and rapid prototypes with the Multi Components Prototype concept (Rivette et al., 2007). This concept aims to decompose a mechanical prototype part on an assembly of several components. There are two main reasons for the multi-components decomposition:

- To include the evolutionary requirement of the prototype regarding to the tests that are performed on it;

- To help designers to choose the best manufacturing process for each component, taking into account time, cost and feasibility of different manufacturing processes.

A major problem in hybrid modular tooling is how to obtain a well-detailed view of the tool manufacturing complexity, in order to create a hybrid modular tool with reduced complexity (and consequently with a lower manufacturing cost). In fact, there are often few details of the tool that can change the manufacturing process choice (a curve radius of a small concave shape for example). This consideration forces the development of a manufacturability analysis with a well-detailed point of view. So manufacturability must be evaluated for the whole tool to discriminate which areas are the most complex to realize. This manufacturability analysis is not easy to perform with a global view, so the tool must be decomposed in smaller parts to help this analysis. 
Many works have been done on manufacturability analysis, especially in the late 1990s, with the Design For Manufacturing (DFM) concept. DFM involves simultaneously considering design goals and manufacturing constraints in order to identify and to alleviate manufacturing problems while the product is being designed; thereby reducing the lead time for product development and improving product quality (Gupta et al., 1997). Most of the studies on DFM methods imply using a feature decomposition of the part CAD model, and associating manufacturability evaluation with each feature. The problem is that features usually rely on one specific field. As an example, machining features are developed for mechanical product definition for process planning (ISO 10303-224), but there are still no manufacturing features for additive process. For free-form surfaces, usually used in tooling design, machining features do not bring enough information on the shape. Furthermore, comparing different manufacturing processes would involve including different manufacturing features which would make the evaluation of manufacturing complexity difficult. So the hybrid modular tooling manufacturability analysis cannot be based on a feature decomposition, and another tool CAD model decomposition method has to be developed.

\section{Related works}

\subsection{Examples of CAD model decompositions}

Each solid modeling method (CSG, B-rep, decomposition method) has its advantages and disadvantages relative to the others in term of accuracy, robustness, data structure and computing time.

Construction Solid Geometry (CSG) method is very popular because this method can complete Boolean operations of any 3D part model relatively easily and accurately. The problem in the CSG approach is that it is computationally expensive to represent the parts with irregular surfaces (Tarng and Chang, 1993).

A common decomposition method used in layered manufacturing is stereolithography (STL) format. A major problem with STL is on its representation of curved surface, which can only be approximated by triangular facets (Kumar and Dutta, 1997). The manufacturing complexity analysis will not be based on this format, because even if this deviation can be controlled according to user's requirements on approximation accuracy, information is lost.

\subsection{Concept of the octree model}

An octree is a tree data structure, which represents a three-dimensional object by the division of space into small cubic boxes, or small parallelepipeds. The size of each box depends on the local geometric complexity of the object represented (Kim, 1998). Each box in space corresponds to a node in the tree and each node is referred to as an octant. To explain how octree decomposition is constructed, Figure 2 shows an example of a 2-D object decomposed in quadtree.

Take in Figure 2.

To construct an octree, the object is first enclosed by the smallest box (octant) that can completely contain the object in any direction. This octant (a cube or a parallelepiped) makes up the root level of the octree. It is then subdivided into 8 sub-octants (4 sub-octants in case of a two-dimensional object) which then represent the first level. The octants are classified into three categories: black (full), white (empty) and grey (partially filled). Black octants are those that are completely contained in the object of interest, whereas white ones are those that are completely outside the object. Grey octants are those 
that are partially inside and outside the object. The subdivision process is performed on grey octants until a desired resolution is reached. The specified accuracy is used to determine the final size of the smallest octants (Ding et al., 2004).

Octree decompositions have been used for several years, first in computer graphics (Szeliski, 1990). In mechanical engineering, octree decomposition is used for the verification of numerical command tool paths (Kim, 1998), for interference detection in five axis machining (Ding et al., 2004) and in robotics (Wenger and Chablat, 1997). In rapid prototyping, octree decompositions of 3D models have been used to realize approximate prototypes before final machining (Medellin et al., 2006).

The advantages of using an octree decomposition model are:

- It does not rely on one specific manufacturing process;

- Decomposition model can acquire relatively high accuracy;

- The special location of an octant is determined by an index code; with these codes, the position of each octant could be easily found and the geometric information such as centre point and edge lengths could thus be calculated.

That is why an octree structure is used for the hybrid modular tooling manufacturability analysis.

\section{Manufacturing complexity evaluation}

In this paper, manufacturability indexes are developed to determine which areas of the tool will be the most difficult-to-manufacture, directly from tool CAD model. The most difficult-to-manufacture areas are:

- The areas which will impose an increasing manufacturing time;

- The areas which will increase overall tool cost;

- The areas for which it will be difficult to achieve a high quality level.

Examples of complex-to-manufacture areas:

- The areas with a fine roughness;

- The back drafted areas, which obligate particular milling tool orientations (in five axis machining) or building support (in layered manufacturing).

The aim of this section is to determine several parameters which have a great influence on the time, cost and quality. These parameters provide information on the most difficult-to-manufacture pieces, or areas of a piece.

Three categories can be distinguished: geometric parameters, material information and specifications. The following list of parameters is limited to those which can be determined only with CAD model. So parameters that require a complete manufacturing preparation analysis (for example: cutting-tool path strategy) are not taken into account to be free from manufacturer skills.

\subsection{Geometric parameters}

First of all, the geometry and dimensions of the part to realize clearly affect the manufacturing time, cost and quality. The geometric parameters have not the same influence in case of a subtractive or an additive process.

If the tool is machined, the geometric parameters that lead a mechanical part difficult-to-machine are:

- Maximal dimensions: a given machine has its own limitations on each axis;

- Minimal dimensions: if some dimensions are too small, it will be impossible to machine with traditional milling tools; 
- Slenderness: parts with a high slenderness ratio will be more difficult-to-machine than other ones;

- Geometrical accessibility for the milling tool: machining the bottom of a depth pocket implies using a long milling tool which can generate a bad quality surface;

- Curvature radius: a concave surface with small curvature radius implies using a milling tool with a corresponding radius;

- Back drafted areas: surface orientations sometimes obligate particular milling tool orientations and five-axis machining;

- Free form surface: lots of changes in the surface orientations have a large influence on the number of feed rate alteration;

- Blank volume: the blank dimensions have an impact on the chip quantity and so a consequence on the part cost;

- Etc.

In case of layered manufacturing, other geometric parameters are taken into account to evaluate manufacturing complexity:

- Volume and height: direct influence on manufacturing time;

- Surfaces orientations: the quantity of support has an impact on the material cost, manufacturing time and surface quality;

- Distance from the centre of the platform: the dimensional error strongly depends on the distance from the platform centre (Pessard et al., 2007);

- Area of skin surface: manufacturing time is higher for skin surfaces than for inner surfaces;

- Maximal and minimal dimensions, slenderness: same impact than for a machining process;

- Etc.

\subsection{Material information}

Obviously, the mechanical characteristics of material directly affect manufacturing process parameters.

As an example, when the material to machine is very hard (50-60 hardness Rockwell scale C), a special range of cutting tool materials is required (ceramic metal composites, polycrystalline cubic boron nitride) with low feed rate (Coldwell et al., 2003).

So manufacturability indexes based on material will be defined according to the following characteristics:

- Hardness;

- Young modulus;

- Ductility;

- Microstructure;

- Thermal conductivity;

- Etc.

And in layered manufacturing, the material choice is limited by the different powders available in a machine, and melting point temperature is clearly significant.

\subsection{Technical specification}

The specification of high degree tolerances and surface finish always increase the number of operations required and more expensive machines. 
Of course, the consequence is a rise in the difficulty of manufacturing. Four parameters are very sensitive with respect to the accuracy and dynamical capability of manufacturing equipment (in case of machining process or layered manufacturing process) (Korosec et al., 2005):

- Dimensional tolerance;

- Geometric tolerance;

- Location tolerance;

- Surface finish.

\section{Manufacturability indexes}

These three categories of parameters allow defining two types of manufacturability indexes: global indexes and local indexes. Global indexes are defined for the whole tool. As an example, an index may be calculated from the parameter "Volume". In fact, volume has a great impact on manufacturing time in an additive manufacturing process. Local indexes are defined for each area of the tool, based on an octree decomposition.

Concentration is first put on geometric parameters. Manufacturability indexes have been developed, according to the previous list of parameters. Machinability indexes are defined and presented in Table 1, and layered manufacturability indexes in Table 2.

Take in Table 1.

Take in Table 2.

The indexes based on an analysis of the surface orientations of the tool have not yet been developed. All these indexes must be calculated according to the help of the tool CAD model and its octree decomposition, and without a complete manufacturing preparation.

A procedure has been developed to evaluate manufacturing complexity from a tool CAD model (Kerbrat et al., 2008). The work has been carried out on a CAD software (SolidWorks 2007) with Visual Basic language. The interface of this system is presented in Figure 3.

Take in Figure 3.

If the manufacturability index that has been chosen is a global index, the system directly posts the index value. The higher the value of the index is, the more difficult-to-manufacture the tool is.

In case of local indexes, a step of decomposition is done, according to an octree decomposition algorithm. Then the index value is calculated for every grey and black octants and a color map of manufacturing complexity for this index is drawn (with automatic or customized color scale). For each octant, the higher the value of the index is, the more difficult-to-manufacture the fraction of the tool contained in the octant is. If the accuracy of the decomposition is not satisfying (the octants are too big compared to the dimensions of the tool), another level of decomposition is done, only for grey octants. When a sufficient accuracy is reached, the decomposition is stopped. The accuracy of the octree decomposition must be carefully determined, because if it is too high, it will dramatically increase computing time. Nevertheless, it must not be too small with respect to the smallest dimension of the tool. In this paper, four levels of decomposition have been chosen.

The manufacturability indexes defined in Tables 1 and 2 are calculated with the help of the following equations (Equations 1-8):

(1) $C\left(d_{x}\right)=\frac{L X_{\max }}{X_{\text {machine }}}$ 
where $L X_{\max }$ is the maximal dimension of the tool in $\mathrm{X}$-direction, and $X_{\text {machine }}$ is the length of the $\mathrm{X}$-axis of the machine. $C\left(d_{y}\right)$ and $C\left(d_{z}\right)$ are determined with similar equations. The machines and tool orientation in a machine have to be previously established. For further examples, Hermle C30U HSM and EOS 250 Xtend machine are used with z-direction as spindle axis and layer normal orientation.

(2) $C(r)=\frac{L}{D}$

where $L$ is the minimal length of the milling tool that can machine the surface included in the octant and $D$ is the maximal diameter of the milling tool that can machine the surface included in the octant. This index is based on two reports. In most cases, when the milling tool diameter is reduced, machining time increases. Moreover, when the ratio length/diameter of the milling tool increases, the quality of the piece realized is reduced. It corresponds to a diminish of the milling tool stiffness. $L$ is calculated by the difference of height between the top face of the highest octant and the bottom face of the octant for which $C(r)$ is being calculated. $D$ takes into account both curvature radius of concave surface (a small curvature radius limits the diameter of the milling tool that can machine a concave surface) and space between two surfaces which may limit the milling tool diameter (Figure 4).

Take in Figure 4.

(3) $C(b)=L X_{\text {max }} \times L Y_{\text {max }} \times L Z_{\text {max }}$

$C(b)$ represents the volume of the blank part. The higher $C(b)$ is, the more expensive the rough part is and consequently the more difficult-to-machine the tool is considered.

(4) $C(c)=\frac{L X_{\max } \times L Y_{\max } \times L Z_{\max }}{V}$

where $V$ is the volume of the tool. Chips are considered as material lost, so if $C(c)$ has a low value, the tool will not require a large quantity of chips and the tool will be considered as easy-to-machine.

(5) $C(v)=V$

(6) $C(s)=S_{e x t}$

where $S_{\text {ext }}$ is the area of the whole skin surface of the tool.

(7) $C(h)=z-Z_{0}$

where $z$ is the z-coordinate of the centre of gravity of the volume of the tool contained in the octant and $Z_{0}$ is the $\mathrm{z}$-coordinate of the bottom face of the tool.

(8) $C(\rho)=\sqrt{\left(x-X_{0}\right)^{2}+\left(y-Y_{0}\right)^{2}}$

where $x$ and $y$ are the coordinate in the $X$-Y plane of the centre of gravity of the volume of the tool contained in the octant and $X_{0}$ and $Y_{0}$ are the coordinate of the centre of the tool, considering that the tool will be manufactured with its centre exactly at the centre of the platform.

For each local index, a global one may be calculated (Equation 9) for an easier comparison between manufacturability indexes.

(9)

$$
C\left(i_{\text {global }}\right)=\frac{\sum_{j}\left(C\left(i_{\text {local }}\right)_{j} \times V_{j}\right)}{\sum_{j} V_{j}}
$$

where $V_{j}$ is the volume of the fraction of the tool contained in the octant for which $C\left(i_{\text {local }}\right)_{j}$ is calculated. 


\section{Examples of using the manufacturing complexity evaluation system}

\subsection{Comparison of CAD models for one manufacturing process: modular point of view}

These manufacturability indexes allow comparing different tool CAD models, regarding one manufacturing process. The most difficult-to-manufacture areas may be improved with a modular point of view, designing modules in order to decrease the value of manufacturability indexes in these areas.

As an example, a single-piece test-part CAD model is analyzed in term of local and global indexes for subtractive process. Figure 5(a) presents the test-part, which is representative of dies and molds traditionally made by High-Speed Machining, and Table 3 gives the values of machining indexes. For $C(r)$ - tool rigidity index, a map of machining complexity is obtained (Figure 5(b)) and most difficultto-machine areas are thus known.

Take in Figure 5.

This map provides an accurate view of the manufacturing complexity of the test-part.

With the example of machining process and $C(r)$ as manufacturability index, the easiest-tomanufacture areas are those where there are no limitations for the milling-tool diameter $(C(r)=0)$. The most difficult-to-manufacture areas of the test-part are at the bottom of the circular boss, with a small curvature radius and the surface between the two high bosses. Then the aim is to concentrate on these areas to understand why they are complex-to-manufacture, according to this particular index. The difficulty can be due to a small space between two bosses that allows only small diameter milling tools. On the other hand, the difficulty can be due to a high wall that forces milling tools to be long. Alternatively, because there is a small radius on a concave surface that implies using a milling tool with a small radius. The further step of the methodology is to take into account modular point of view, creating modules, manufactured aside and further gathered, to reduce manufacturing complexity in the previous most difficult-to-machine areas, as it can be seen in Figure 6. In this example, assembly process is not treated.

Take in Figure 6.

For a global comparison of the two CAD models, manufacturability indexes are calculated for the single-piece test-part and the two modules of the modular one (Table 3). Concerning the modular testpart, total indexes are calculated with the following equation (Equation 10).

(10) $C\left(i_{\text {total }}\right)=\frac{C\left(i_{\text {mod.1 }}\right) \times V_{\text {mod.1 }}+C\left(i_{\text {mod. } 2}\right) \times V_{\text {mod.2 }}}{V_{\text {mod.1 }}+V_{\text {mod.2 }}}$

In this methodology, it is still impossible to compare different indexes between themselves, so a comparison of the evolutions of the values of the machinability indexes is done between the two tools (Table 3).

Take in Table 3. 
So it can be concluded that the modular point of view provides a modular tool with less manufacturing complexity because the tool rigidity index $C(r)$ decreases by $40 \%$ whereas the other indexes evolutions are not significant.

\subsection{Comparison of manufacturing processes: hybrid point of view}

Another way of using this system is for comparison of two manufacturing processes (additive and subtractive) for one tool CAD model, in order to determine which parts of the tool may advantageously be machined or realized by a layered manufacturing process.

This second example is based on the comparison of the same test-part, but with changes in the pocket dimensions. The test-part presented in figure 5a has the following pocket dimensions:

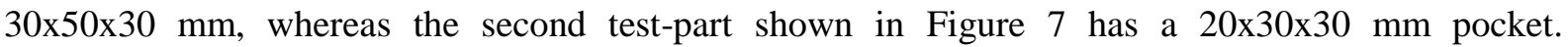
Manufacturability indexes are calculated, first for machining process (Figure 8).

Take in Figure 7.

Take in Figure 8.

An analysis of the evolutions in machinability indexes between the two CAD models is done. In this example, it can be seen that changing the dimensions of the test-part provide new areas of the second test-part very complex-to-machine, according to the $C(r)$ index. The $C\left(r_{\max }\right)$ value is doubled and the $C\left(r_{\text {global }}\right)$ value increases by $22 \%$, whereas the other values evolutions are not significant.

Then, manufacturability indexes are calculated for additive process (Figure 9).

Take in Figure 9.

Changing the dimensions of the test-part provide few evolutions in the values of the different indexes. So the two test-parts will have the same level of manufacturing complexity in case of an additive process.

Consequently, for this second test-part, the areas which are the most complex-to-machine would advantageously be manufactured with an additive process, creating a hybrid part. In this hybrid part, the areas which are easy-to-machine would be machined and the most difficult-to-machine would be manufactured by a layered manufacturing process.

\section{Conclusion and future work}

In this paper, a new manufacturing complexity evaluation system is exposed. Manufacturability indexes have been developed, an interface is created to calculate them directly from a tool CAD model.

This approach provides an accurate view of which parts of the tool have to be improved in order to reduce manufacturing difficulties. Then modular and hybrid points of view allow designing a hybrid modular tool which will be less difficult-to-machine than the first single-piece tool. Two simple examples have been treated to illustrate the possibilities of this new methodology. In the first example, the massive decrease of the local index value is exploited to choose the modular tool design instead of the traditional single-piece tool design. The second example shows a typical case in which the hybrid modular concept brings opportunity to manufacture a piece with both additive and subtractive processes. 
In this paper, the modular point of view has been utilized for decreasing manufacturing complexity, and may also been applied for increasing flexibility of the tool. This topic is not treated here and will be integrated in the system later. To have a more detailed view of manufacturing complexity, more accurate manufacturability indexes may be calculated, with other parameters involved and organized with fuzzy logic, so further researches will be conducted to develop new manufacturability indexes (based on material information and specifications). Further studies have to be done in order to be able to compare different indexes between themselves and to take into account the assembly constraints generated by a hybrid modular design.

\section{References}

Coldwell H., Woods R., Paul M., Koshy P., Dewes R. and Aspinwall D. (2003), "Rapid machining of hardened AISI H13 and D2 moulds, dies and press tools", Journal of Materials Processing Technology, Vol. 135, pp. 301-311.

Ding S., Mannan M.A. and Poo A.N. (2004), "Oriented bounding box and octree based global interference detection in 5-axis machining of free-form surfaces", Computer-Aided Design, Vol. 36, pp. 1281-1294.

Gupta S.K., Das D., Regli W.C. and Nau D.S. (1997), "Automated manufacturability analysis: A survey", Research in Engineering Design, Vol. 9 No. 3, pp. 168-190.

Kerbrat O., Mognol P. and Hascoet J.Y. (2008), "Manufacturing complexity evaluation for additive and subtractive processes: Application to hybrid modular tooling", $19^{\text {th }}$ Solid Freeform Fabrication Symposium, Austin, Texas, USA, August 4-6.

Kim J. (1998), "NC verification using octree", MSc thesis, MIT, Cambridge, MA.

Korosec M., Balic J. and Kopac J. (2005), "Neural network based manufacturability evaluation of free form machining", International Journal of Machine Tools \& Manufacture, Vol. 45, pp. 13-20.

Kumar V. and Dutta D. (1997), "An assessment of data formats for layered manufacturing", Advances in Engineering Software, Vol. 28, pp.151-164.

Medellin H., Corney, J., Davies J.B.C., Lim T. and Ritchie J.M. (2006), "Algorithms for the physical rendering and assembly of octree models", Computer-Aided Design, Vol. 38, pp. 69-85.

Mognol P., Hascoët J.Y., Rivette M. and Kerbrat O. (2007), "Evaluation of Hybrid Tooling with HSM-EDM-DMLS: methods for comparison of time and cost using product examples", EurouRapid2007, Frankfurt, Germany, December, 3-4, pp.179-188.

Pessard E., Mognol P., Hascoët J.Y. and Gerometta C. (2007), "Complex cast parts with rapid tooling: rapid manufacturing point of view", The International Journal of Advanced Manufacturing Technology, Vol. 39 No. 9/10, pp 898-904.

Rivette M., Hascoët J.Y. and Mognol P. (2007), "A based-graph methodology for hybrid rapid design”, Journal of Engineering Manufacture, Vol. 221 No 4, pp 685-697.

Szeliski R. (1990), "Real-time octree generation from rotating objects. Technical Report, Cambridge Research Laboratory.

Tarng Y.S. and Chang W.S. (1993), "Dynamic NC simulation of milling operations", Computer-Aided Design, Vol. 25 No. 12, pp 769-775.

Wenger P. and Chablat D. (1997), "Definition sets for the Direct Kinematics of Parallel Manipulators", $8^{\text {th }}$ International Conference in Advanced Robotics, Monterey, USA, pp. 859-864. 
Figure 1

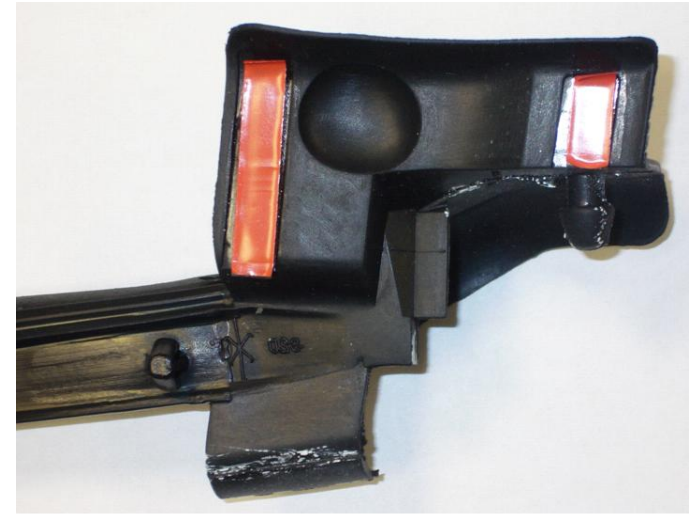

(a) Seal rear door of a vehicle

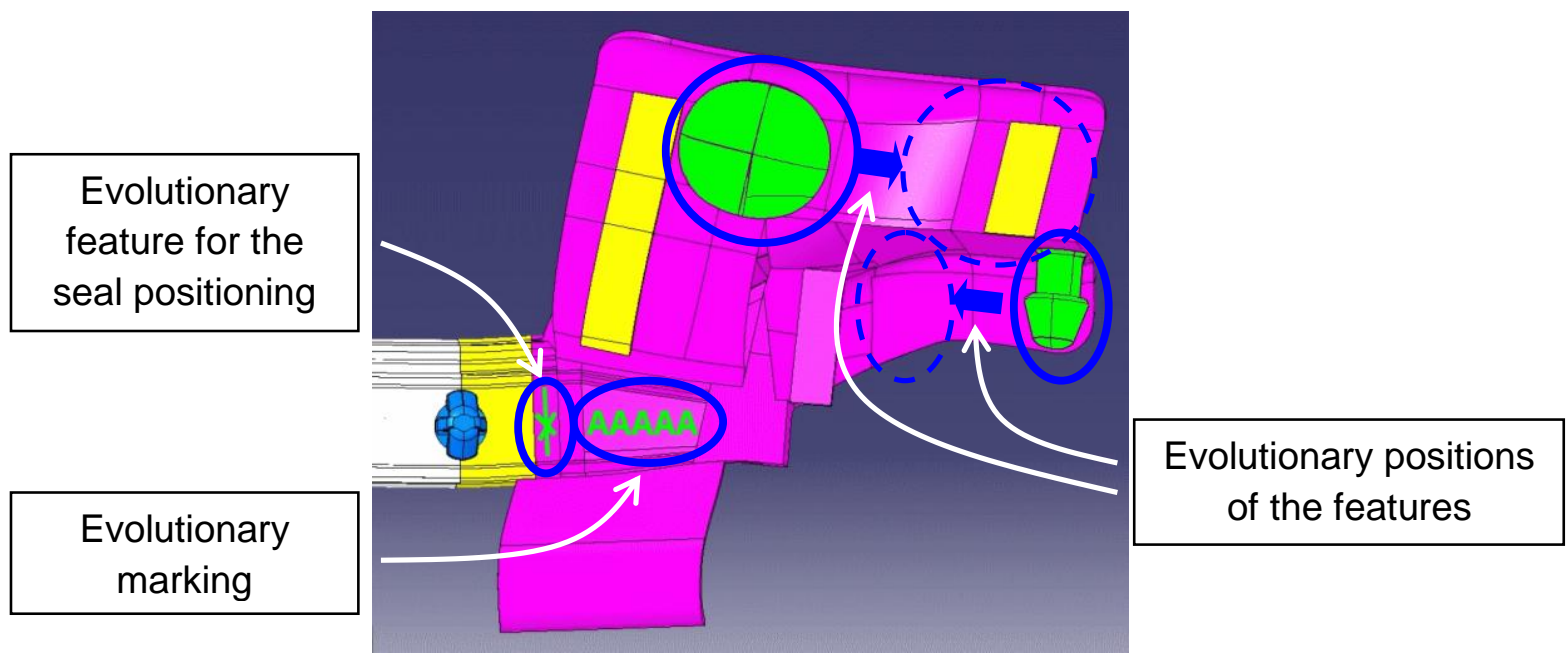

(b) Seal rear door CAD model with evolutionary areas

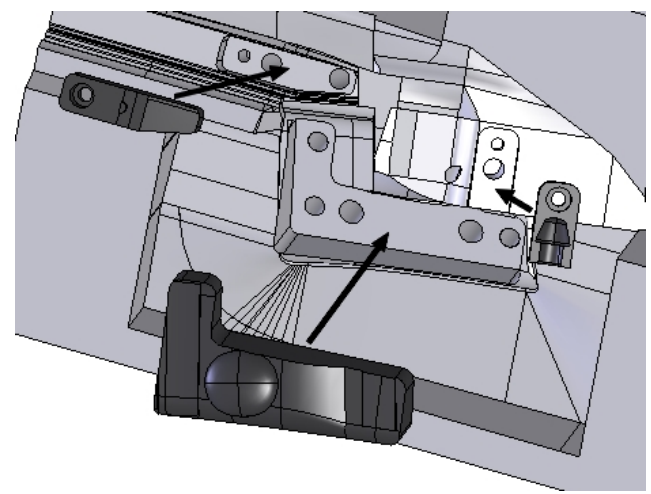

(c) Seal rear door hybrid modular tool CAD model

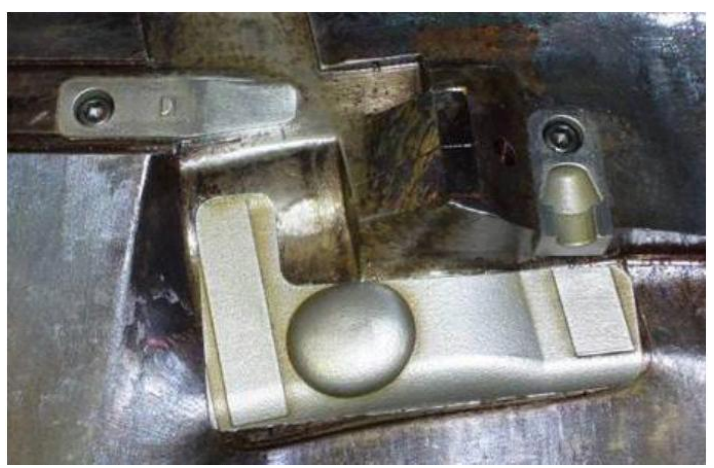

(d) Seal rear door hybrid modular tool 
Figure 2

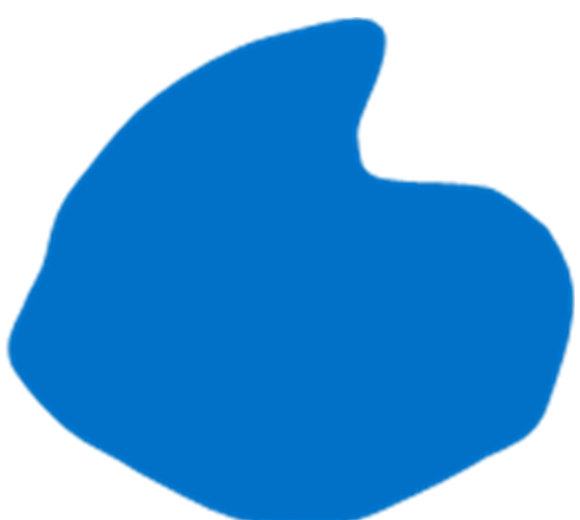

(a) Shape to decompose

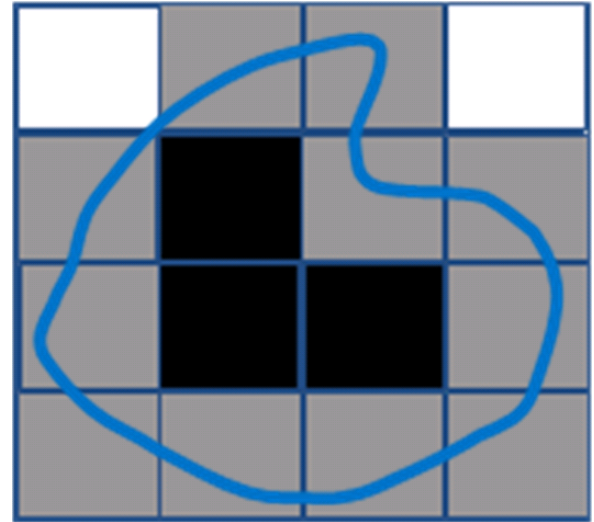

(b) Level 2 of decomposition

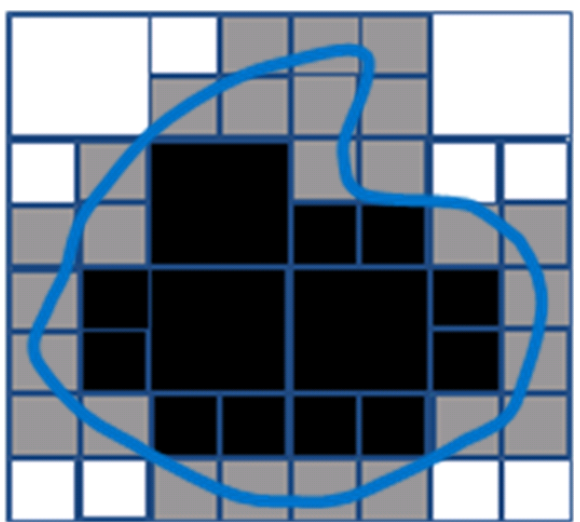

(c) Level 3 of decomposition

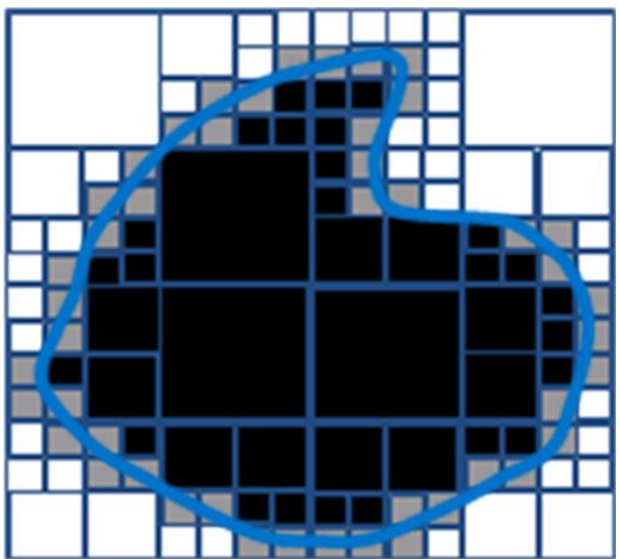

(d) Level 4 of decomposition 
Figure 3

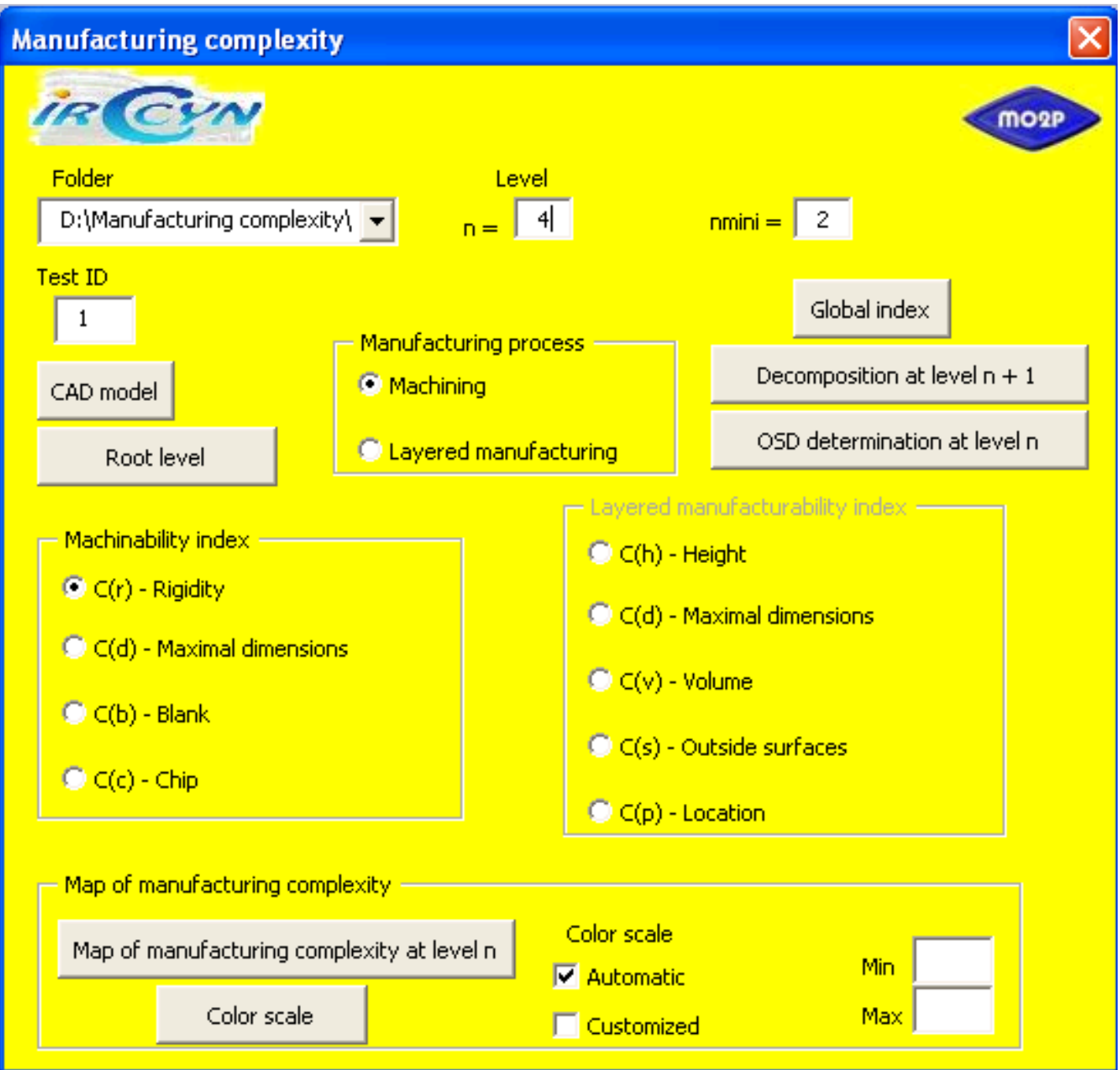


Figure 4

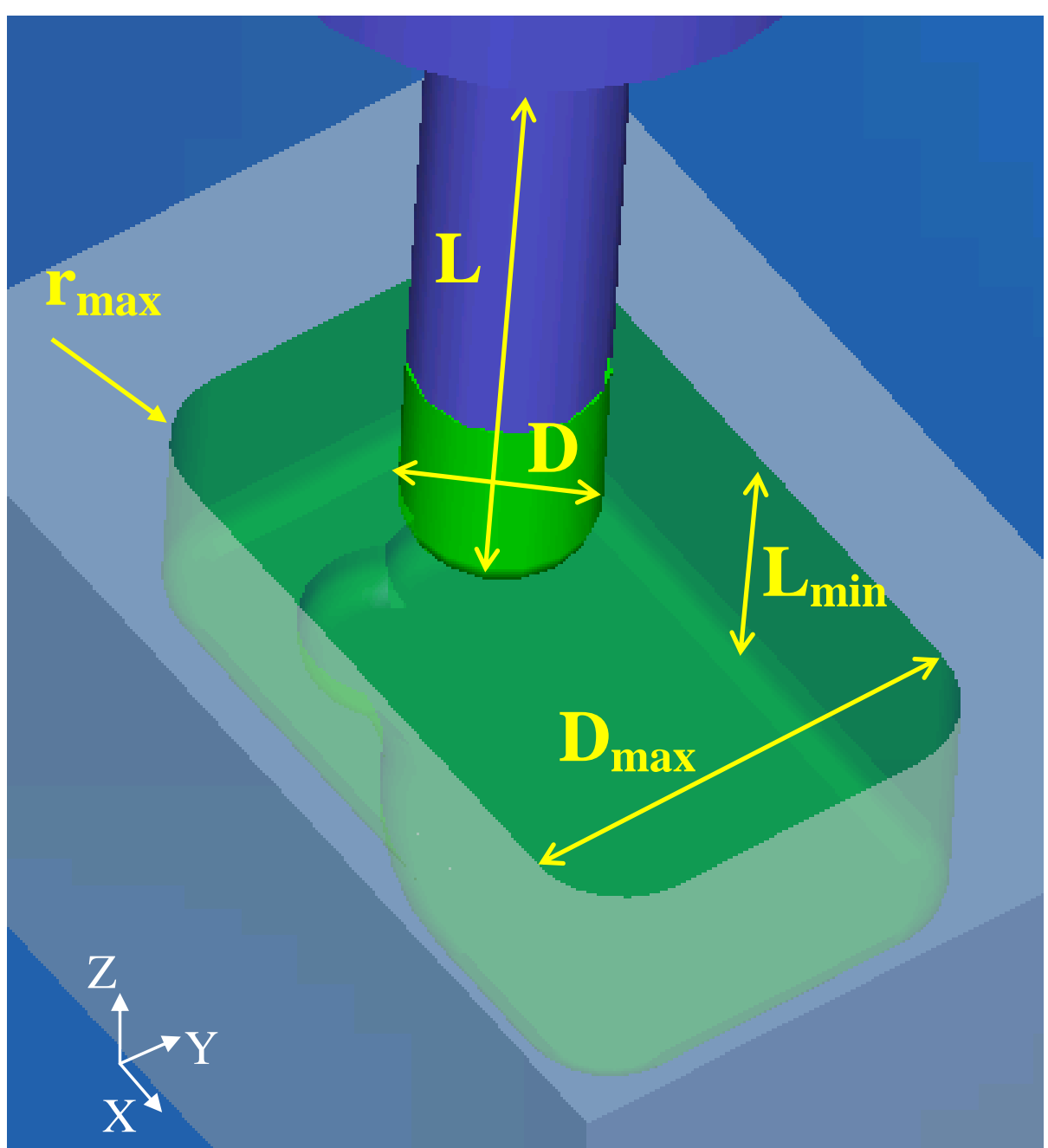


Figure 5

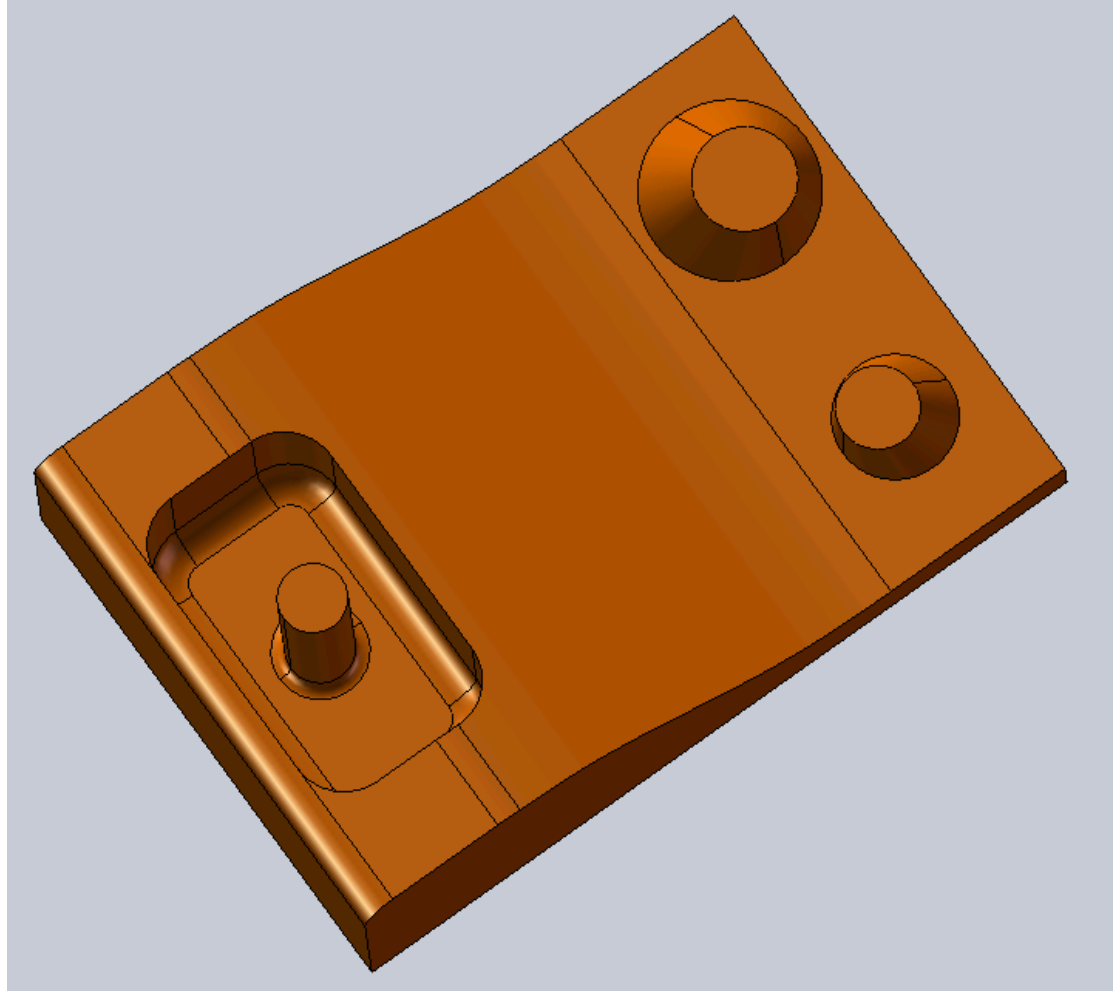

(a) CAD model

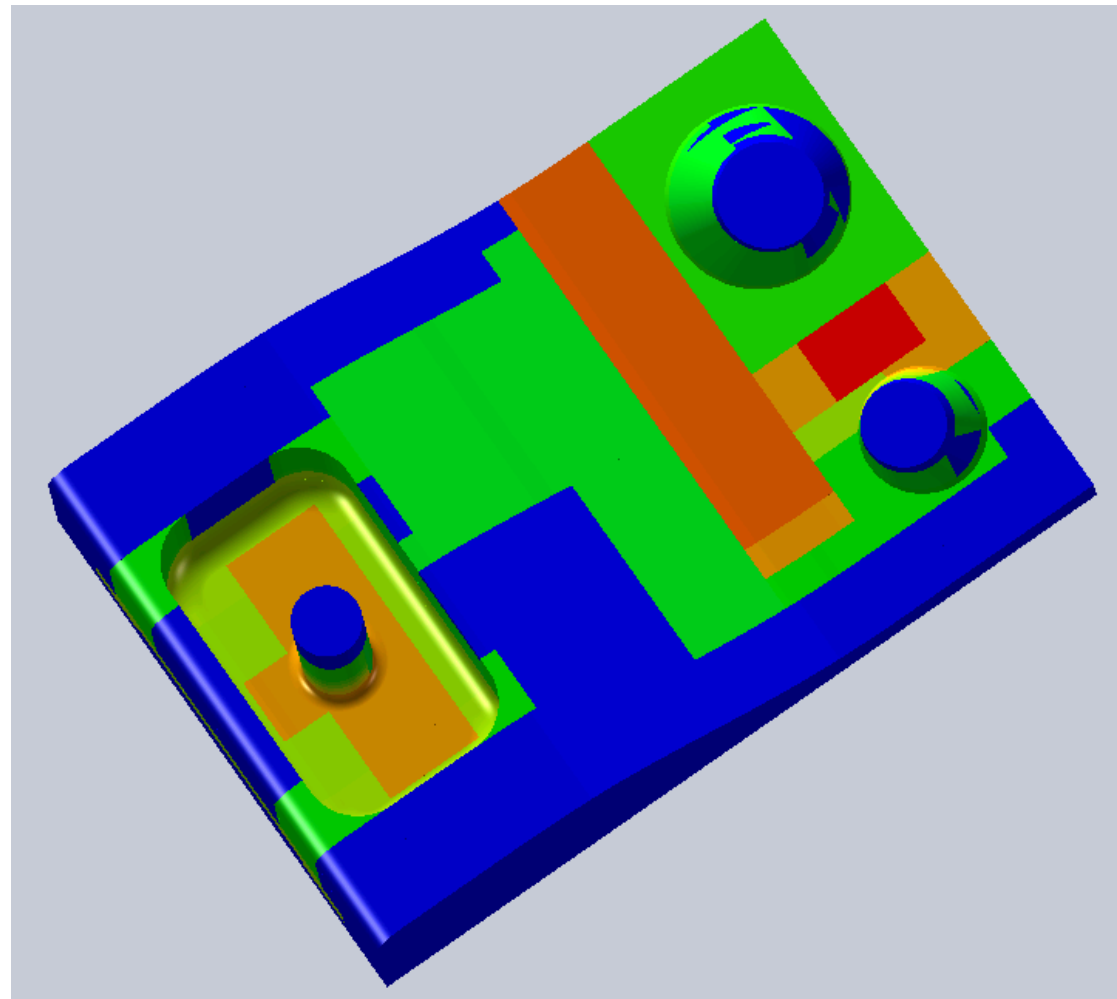

(b) Map of manufacturing complexity

$\begin{array}{lllllllllllllllll}0.00 & 1.90 & 2.00 & 2.03 & 2.15 & 2.17 & 2.19 & 2.34 & 2.60 & 2.70 & 2.71 & 2.75 & 2.86 & 3.13 & 3.39 & 3.65 & 4.06\end{array}$ (c) Color scale 
Figure 6

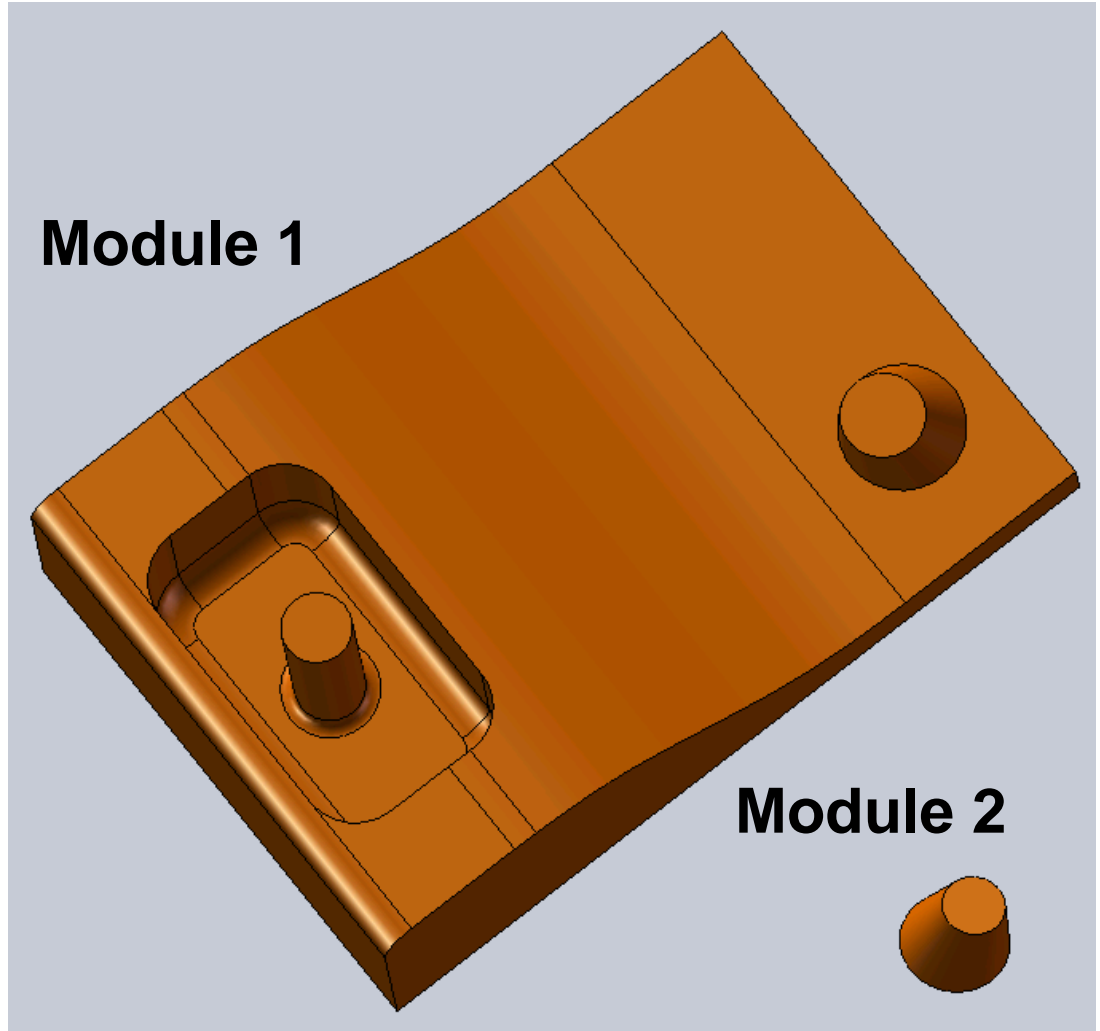

(a) CAD model

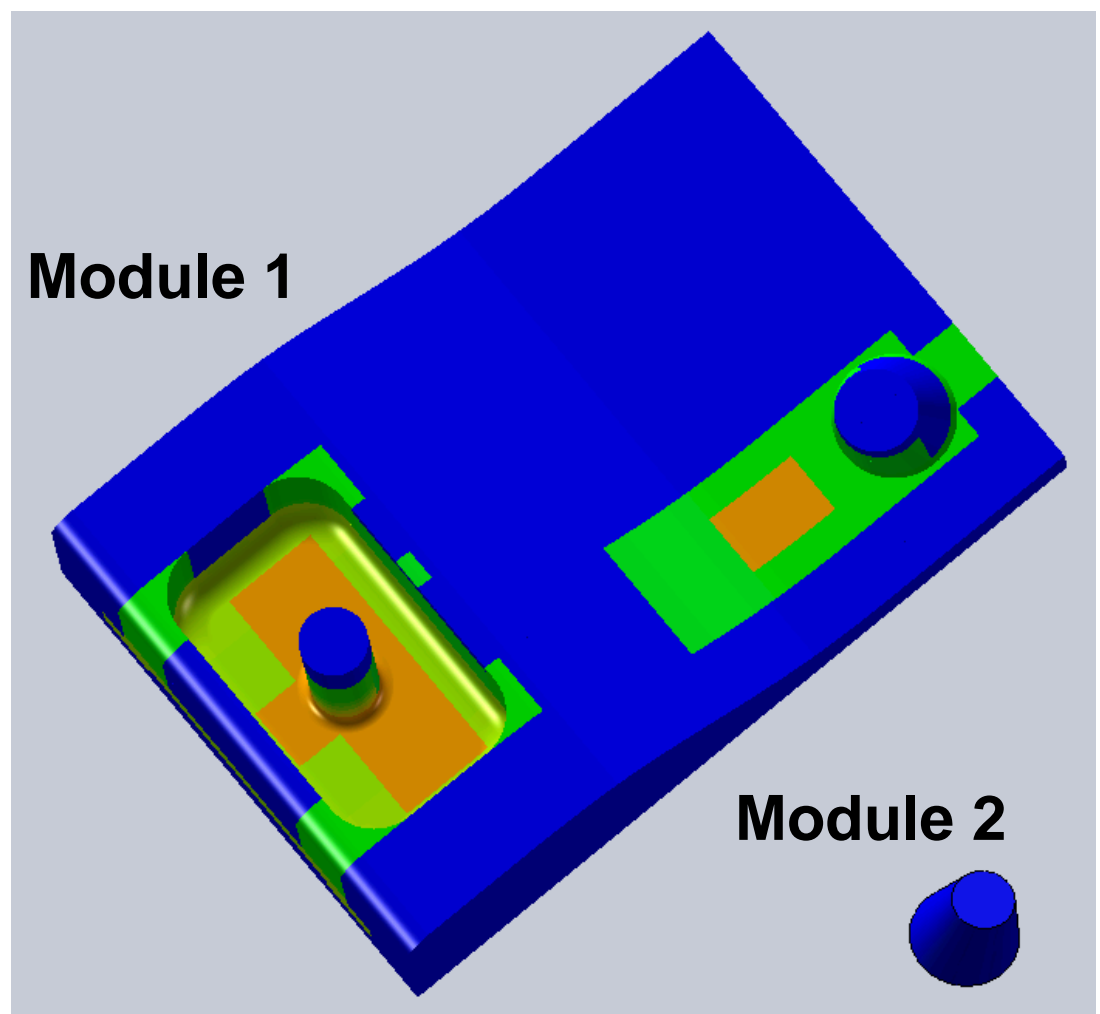

(b) Map of manufacturing complexity 
Figure 7

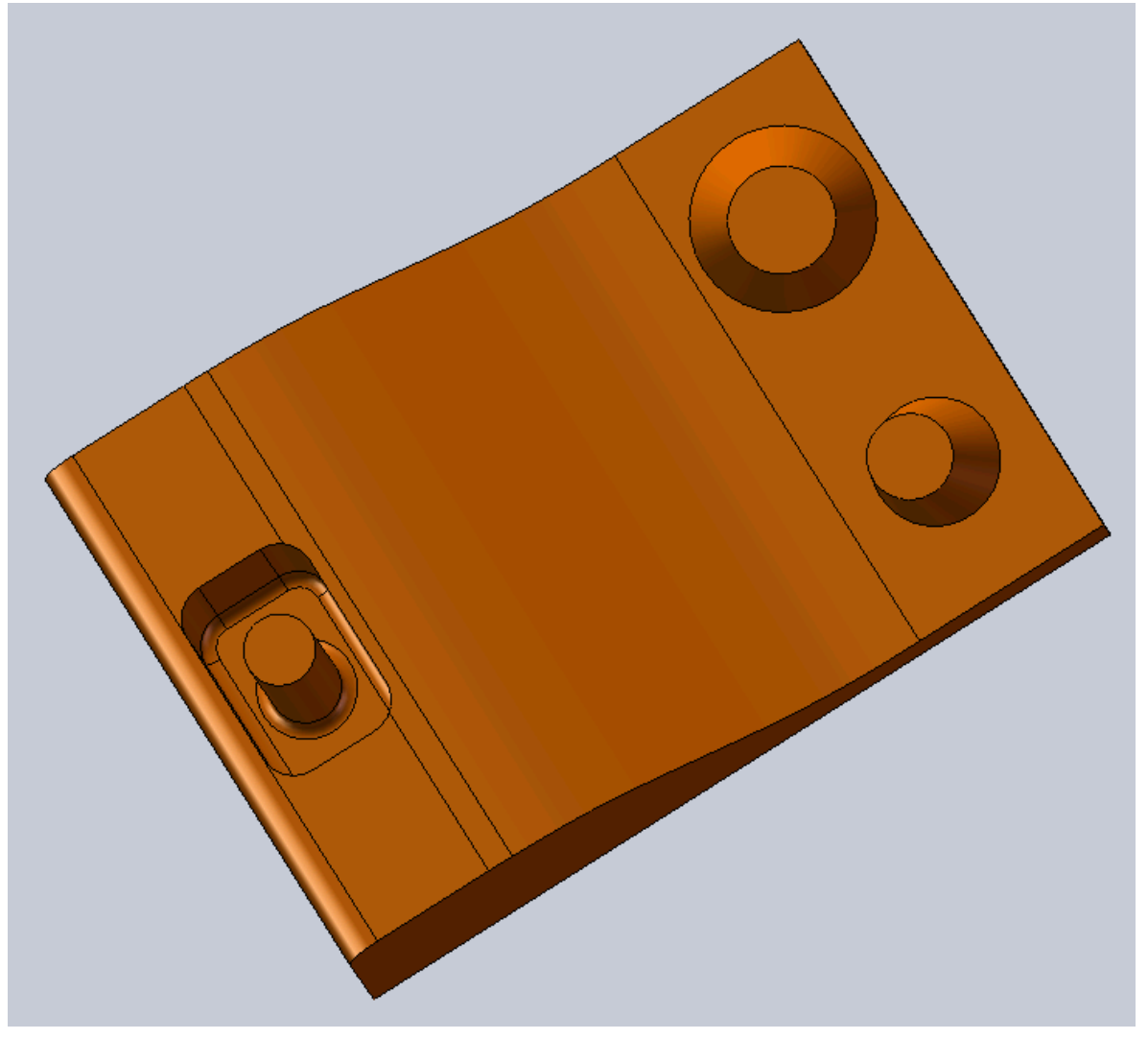


Figure 8

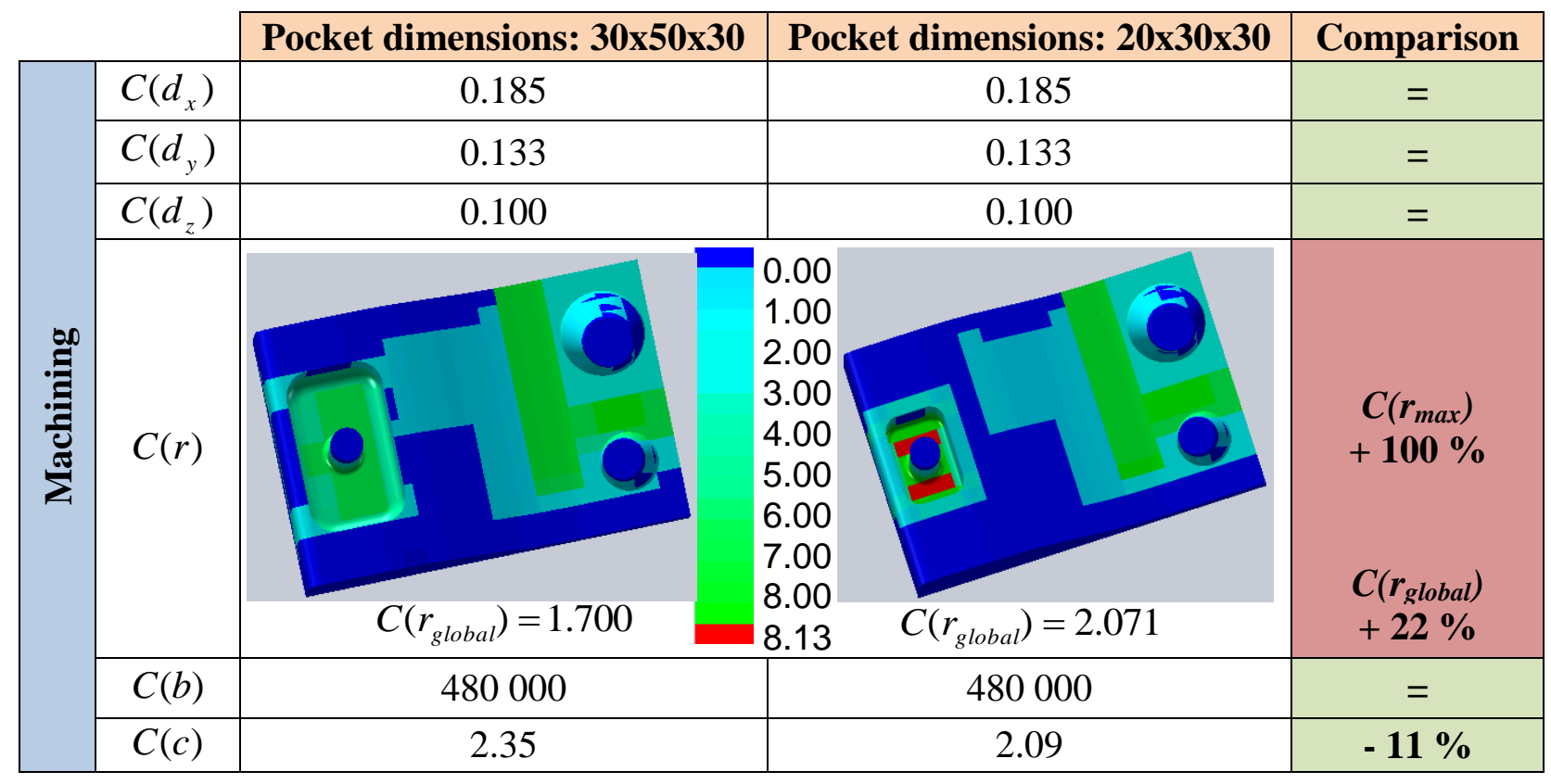


Figure 9

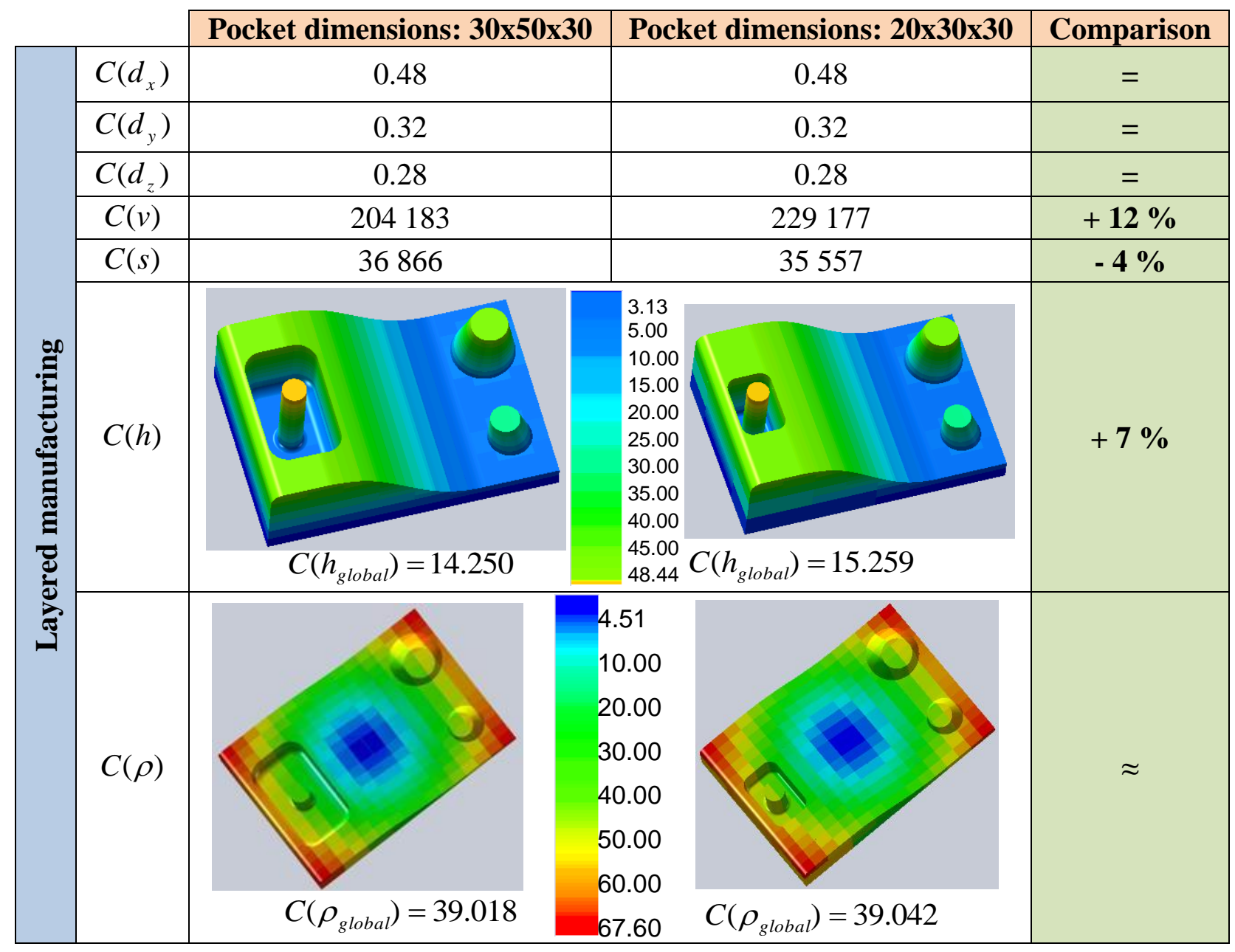


Table 1

\begin{tabular}{|c|c|c|}
\hline Index & Linked to & Type \\
\hline$C\left(d_{x}\right) C\left(d_{y}\right) C\left(d_{z}\right)$ & Maximal dimensions & Global \\
\hline$C(r)$ & Tool rigidity & Local \\
\hline$C(b)$ & Blank volume & Global \\
\hline$C(c)$ & Chip quantity & Global \\
\hline
\end{tabular}

Table 2

\begin{tabular}{|c|c|c|}
\hline Index & Linked to & Type \\
\hline$C\left(d_{x}\right) C\left(d_{y}\right) C\left(d_{z}\right)$ & Maximal dimensions & Global \\
\hline$C(v)$ & Volume & Global \\
\hline$C(s)$ & Skin surface & Global \\
\hline$C(h)$ & Height & Local \\
\hline$C(\rho)$ & Distance from the centre of the platform & Local \\
\hline
\end{tabular}

Table 3

\begin{tabular}{|c|c|c|c|c|c|}
\cline { 2 - 5 } \multicolumn{1}{c|}{} & \multirow{2}{*}{ Single-piece test part } & \multicolumn{3}{c|}{ Modular test-part } & \multirow{2}{*}{ Comparison } \\
\cline { 2 - 5 } & & Module 1 & Module 2 & Total & \\
\hline$C\left(d_{x}\right)$ & $\mathbf{0 . 1 8 5}$ & 0.185 & 0.043 & $\mathbf{0 . 1 7 8}$ & $\mathbf{- 4} \%$ \\
\hline$C\left(d_{y}\right)$ & $\mathbf{0 . 1 3 3}$ & 0.133 & 0.043 & $\mathbf{0 . 1 2 9}$ & $\mathbf{- 3} \%$ \\
\hline$C\left(d_{z}\right)$ & $\mathbf{0 . 1 0 0}$ & 0.100 & 0.06 & $\mathbf{0 . 0 9 8}$ & $\mathbf{- 2} \%$ \\
\hline$C\left(r_{\text {global }}\right)$ & $\mathbf{1 . 7 0 0}$ & 0.998 & 0 & $\mathbf{0 . 9 9 8}$ & $\mathbf{- 4 0} \%$ \\
\hline$C(b)$ & $\mathbf{4 8 0 0 0 0}$ & 480000 & 20280 & $\mathbf{4 5 7 2 7 3}$ & $\mathbf{- 5} \%$ \\
\hline$C(c)$ & $\mathbf{2 . 3 5}$ & 2.09 & 2.01 & $\mathbf{2 . 0 9}$ & $\mathbf{- 1 1} \%$ \\
\hline
\end{tabular}

